\title{
MAGEA4 induces growth in normal oral keratinocytes by inhibiting growth arrest and apoptosis
}

\author{
SHEETAL BHAN ${ }^{1}$, ALICE CHUANG ${ }^{2}$, SANDEEP S. NEGI ${ }^{2}$, CHAD A. GLAZER ${ }^{1}$ and JOSEPH A. CALIFANO ${ }^{1-3}$ \\ Departments of ${ }^{1}$ Otolaryngology - Head and Neck Surgery, ${ }^{2}$ Oncology, Johns Hopkins Medical Institutions; \\ ${ }^{3}$ Milton J. Dance Head and Neck Center, Greater Baltimore Medical Center, Baltimore, MD, USA
}

Received May 31, 2012; Accepted June 25, 2012

DOI: 10.3892/or.2012.1934

\begin{abstract}
Cancer testis antigens (CTAs) are proteins that are normally expressed only in male germ cells and are aberrantly upregulated in a variety of cancers such as melanomas and lung cancer. MAGEA proteins belong to Class I CTAs and are being utilized as targets for cancer immunotherapy. Despite the discovery of the first CTA (MAGEA1) 20 years ago, the functions of these proteins remain poorly understood and evidence suggests both oncogenic as well as tumor suppressive roles for these proteins. Herein, we investigated the role of MAGEA4 in promoting cell growth. When overexpressed, MAGEA4 promotes growth of spontaneously transformed normal oral keratinocytes (NOK-SI). To understand the mechanism of growth stimulation by MAGEA4, we explored the effect of overexpressing MAGEA4 on cell cycle and apoptosis. MAGEA4 inhibits growth arrest of cells in the G1 phase of the cell cycle. We also found that overexpression of MAGEA4 inhibits G418-induced apoptosis of NOK-SI cells. Interestingly, this inhibition was accompanied by repression of two p53 downstream genes, $B A X$ and $C D K N 1 A$. Our results indicate that MAGEA4 promotes growth by preventing cell cycle arrest and by inhibiting apoptosis mediated by the p 53 transcriptional targets.
\end{abstract}

\section{Introduction}

Cancer testis antigens (CTAs) are proteins that are normally expressed only in the male germ cells. These proteins are not expressed in other normal somatic tissues but are aberrantly upregulated in a variety of cancers (1-3). CTAs are divided into two categories, X-chromosome encoded (CT-X) and non X-chromosome encoded. To date, 110 CTAs have been

Correspondence to: Dr Joseph A. Califano, Department of Otolaryngology - Head and Neck Surgery, Johns Hopkins Medical Institutions, 1550 Orleans Street, Room 5N.04, Baltimore, MD 21231, USA

E-mail: jcalifa@jhmi.edu

Key words: MAGEA4, cancer testis antigens, head and neck squamous cell carcinoma reported in the literature of which, $\sim 30$ are encoded by multigene families on the X-chromosome also called CT-X genes $(1,4)$. Amongst the CT-X is the MAGE family whose members are divided into two classes. MAGE-A, -B and -C have been classified as class $I$ and the remaining MAGE genes are classified as class II (5). The non X-chromosome encoded CTAs are distributed throughout the genome and do not form multigene families. Several studies have evaluated the expression of CTAs to show that these proteins are frequently expressed in variety of cancers. Melanomas, lung cancers and ovarian cancers have the highest frequency of CTA expression while leukemia, lymphomas, renal, colon and pancreatic cancers have a lower frequency of CTA expression. The frequency of expression of these proteins has also been found to correlate with tumor grade and metastatic behavior. An expression array study performed in lung cancer cell lines showed that $30 \%$ of the overexpressed genes (6 out of 20) were CTAs (5 MAGEA and $N Y$-ESO-1) (6).

CTAs are known to be regulated epigenetically in cancers (7-9). Derepression of these genes in cultured cells after treatment with DNA methyl-transferase inhibitors like 5-aza-2'-deoxycytidine shows that demethylation is a key factor governing the regulation of these genes (9-11). Using an integrative epigenetic screening approach, we have previously shown that CTAs are upregulated in non-small cell lung cancer (NSCLC) and head and neck squamous cell carcinoma (HNSCC) by promoter hypomethylation $(10,11)$. Recently, we showed that transcription factor BORIS (brother of the regulator of imprinted sites) regulates 3 MAGEA genes, MAGEA2, A3 and $A 4$, by binding to their promoters and enriching transcription activating histone modifications (12). Two earlier reports have also implicated BORIS in the activation of MAGEAI and NY-ESO-1 genes $(13,14)$.

Because of their exclusive expression in a wide variety of human cancers and their ability to elicit cellular and humoral immune responses, CTAs have been explored as potential targets for cancer immunotherapy $(4,15,16)$. Two CTAs, MAGEA3 and NY-ESO-1, are being evaluated as targets for cancer vaccines in multiple clinical trials. Although much attention has been given to the development of cancer vaccines based on these antigenic proteins, their physiological functions remain unclear. Recent efforts to resolve their functional roles provide evidence for both tumor suppressive and oncogenic roles for these proteins. While some studies have shown that MAGEs are involved in 
growth stimulation and apoptosis inhibition (17-21), others have shown that MAGEs are pro-apoptotic $(22,23)$.

In the present study, we investigated the role of MAGEA4 in promoting cell growth and the possible mechanisms by which it acts as a growth promoter. We showed that overexpression of MAGEA4 prevents cell cycle arrest and also makes the cells resistant to apoptosis. Further, we showed that overexpression of MAGEA4 represses p53 targets, $B A X$ and $C D K N 1 A$.

\section{Materials and methods}

Tissue samples and cells. Human tissue samples were collected after approval from Johns Hopkins Medicine Institutional Review Board and informed written consent from the patients. Spontaneously immortalized normal oral keratinocytes (NOKSI) were provided by Dr Silvio Gutkind (National Institutes of Health, Bethesda, MD).

Transfection of human expression vectors and anchoragedependent growth assay. Full-length ORF cDNA of MAGEA4 in pCMV-SPORT6 vector was obtained from Invitrogen (Carlsbad, CA). NOK-SI cells (21) were plated in 96-well plates and transfected with either the MAGEA4 expression vector or the empty vector using FuGene 6 transfection reagent per the vendor's instructions (Roche). Cell Counting kit-8 (CCK-8) (Dojindo) absorbance was measured by the Spectramax M2e 96-well fluorescence plate reader Molecular Devices (Sunnyvale, CA) after transfection every $24 \mathrm{~h}$ for $72 \mathrm{~h}$. Absorbance was also measured at $0 \mathrm{~h}$ (time of transfection) to ensure equal plating of cells.

RNA extraction and quantitative Reverse-Transcription PCR $(q R T-P C R)$. Total RNA was extracted using QIAzol and RNeasy mini kit (Qiagen). RNA was reverse transcribed to cDNA using qScript cDNA mix (Quanta Biosciences). Realtime PCR was performed using the Fast SYBR green master mix on the ABI 7900HT real-time PCR machine (Applied Biosystems). Primers used were: GAPDH, forward 5'-AGC CACATCGCTCAGACAC-3' and reverse 5'-GCCCAATAC GACCAAATCC-3'; MAGEA4, forward 5'-AGGAGAAGA TCTGCCTGTGG-3' and reverse 5'-CAGCCTCCTGCTCCT CAGTA-3'.

Cell cycle assay. For cell cycle analysis, NOK-SI cells were plated in 100-mm dishes and transfected with the MAGEA4 expression vector or empty vector using FuGene HD (Roche). To ensure contact inhibition of growth, cells were allowed to remain confluent for $48 \mathrm{~h}$ before being processed further. The cells were then harvested by trypsinization, fixed in methanol and stained with $0.1 \mathrm{mg} / \mathrm{ml}$ propidium iodide. DNA content was measured using flow cytometric analysis performed using a FACScan flow cytometer (Becton Dickinson).

Apoptosis assay. NOK-SI cells were transiently transfected with MAGEA4 expression vector or the control empty vector. Twenty-four hours post-transfection, the cells were treated with $2.5 \mathrm{mg} / \mathrm{ml} \mathrm{G} 418$ for 2 days to induce apoptosis (24). At the end of 2 days, the cells were harvested by trypsinization. Cell growth media were also collected to ensure collection of floating apoptotic cells. Cells were then treated for the caspase-3 staining using the FITC active caspase-3 apoptosis kit (BD Biosciences) according to the manufacturer's instructions. The stained cells were subjected to flow cytometry and data were analyzed using FlowJo software (Tree Star Inc.).

\section{Results}

MAGEA4 is differentially overexpressed in primary HNSCC tissues. We have previously shown that MAGEA4 is a candidate oncogene derepressed by promoter hypomethylation in HNSCC. In that study, we performed bioinformatics analysis called Cancer Outlier Profiling Analysis (COPA) on microarray data of 49 primary HNSCC and 19 normal mucosal tissues retrieved from the Oncomine database (www.oncomine.org) to show that MAGEA4 was significantly overexpressed in tumors compared to normal tissues. In the present study, we used qRT-PCR to analyze MAGEA4 expression in a separate cohort of 31 HNSCC and 12 normal non-cancer upper aerodigestive mucosal samples. We found significant upregulation of MAGEA4 in the tumor samples compared to the normal samples $(\mathrm{p}<0.005)$ (Fig. 1). Of 31 tumor samples, 24 showed significant expression of MAGEA4.

MAGEA4 induces growth in NOK-SI cells by preventing cell cycle arrest. To elucidate the function of MAGEA4 in HNSCC, we investigated the effect of overexpressing MAGEA4 in a spontaneously transformed normal oral keratinocyte cell line (NOK-SI) (Fig. 2A). Transient overexpression of MAGEA4 in NOK-SI cells resulted in $37 \%$ increase in cell proliferation at 72-h post-transfection (Fig. 2A). We next investigated if the growth stimulation caused by MAGEA4 is a result of changes in the cell cycle. We transiently overexpressed MAGEA4 in NOK-SI cells and allowed them to go into cell cycle arrest by contact inhibition. We allowed the cells to remain confluent for $48 \mathrm{~h}$ to ensure growth arrest by contact inhibition. The cells were then stained with propidium iodide and analyzed by flow cytometry for cellular DNA content. The analysis showed that a significantly smaller percentage of cells overexpressing MAGEA4 were arrested in G1 phase (68\%) compared to cells transfected with the empty vector (85\%) (Fig. 2B). This shows that MAGEA4 is able to inhibit the growth arrest caused by contact inhibition and allows growth under conditions of high confluency. A follow-up by flow cytometry analysis at 9, 12 and $15 \mathrm{~h}$ after release from density arrest, showed that MAGEA4 overexpressing and control cells progressed through the cell cycle similarly (Fig. 2B).

MAGEA4 inhibits apoptosis and suppresses p53 target genes. To gain further understanding of the mechanism of growth promotion by MAGEA4 in NOK-SI cells, we analyzed the effect of MAGEA4 overexpression on apoptosis. We transiently transfected NOK-SI cells with MAGEA4 expression vector or the control empty vector and then induced apoptosis using G418. After 48 h of treatment with G418, a significantly smaller percentage of MAGEA4 expressing cells (14\%) were positive for active caspase-3 compared to the control cells (39\%) indicating that MAGEA4 expression can block apoptosis induced by G418 (Fig. 3). Control cells and cells overexpressing MAGEA4 not treated with G418, were negative for active caspase-3 (Fig. 3). 


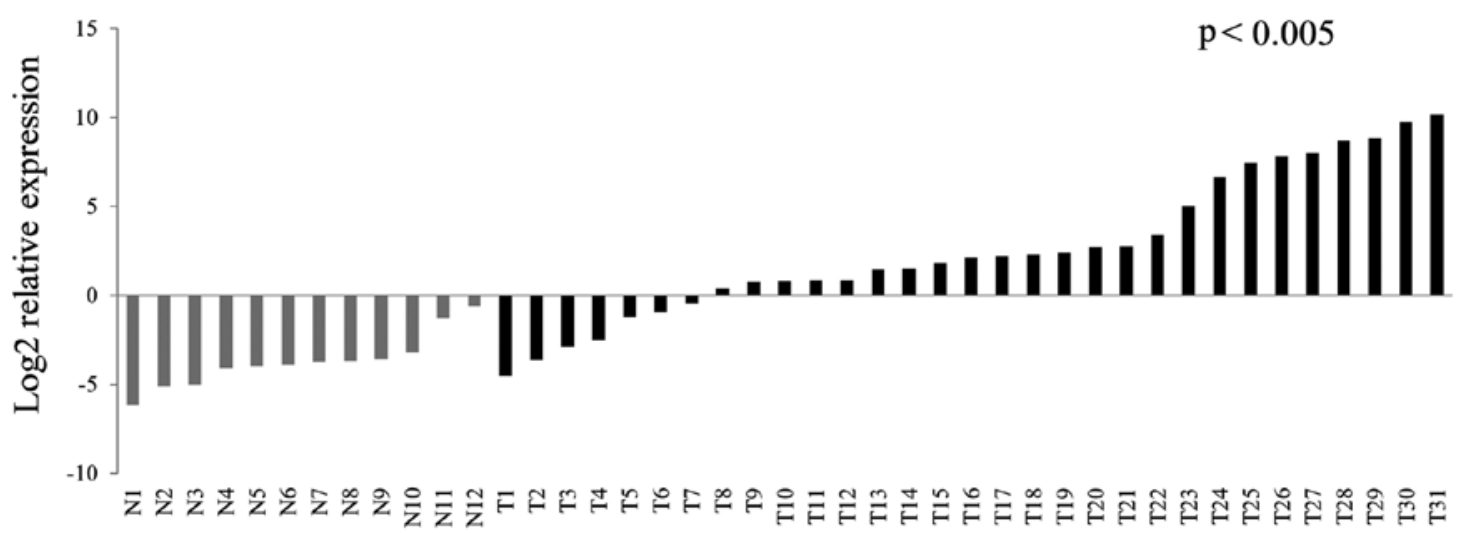

Figure 1. MAGEA4 is differentially overexpressed in HNSCC tissues. Expression of 31 HNSCC tissues (T) and 12 normal mucosa (N) was measured using qRT-PCR. Of 31 tumor samples, 24 showed significant overexpression of MAGEA4 $(\mathrm{p}<0.005)$. Expression was calculated relative to GAPDH.
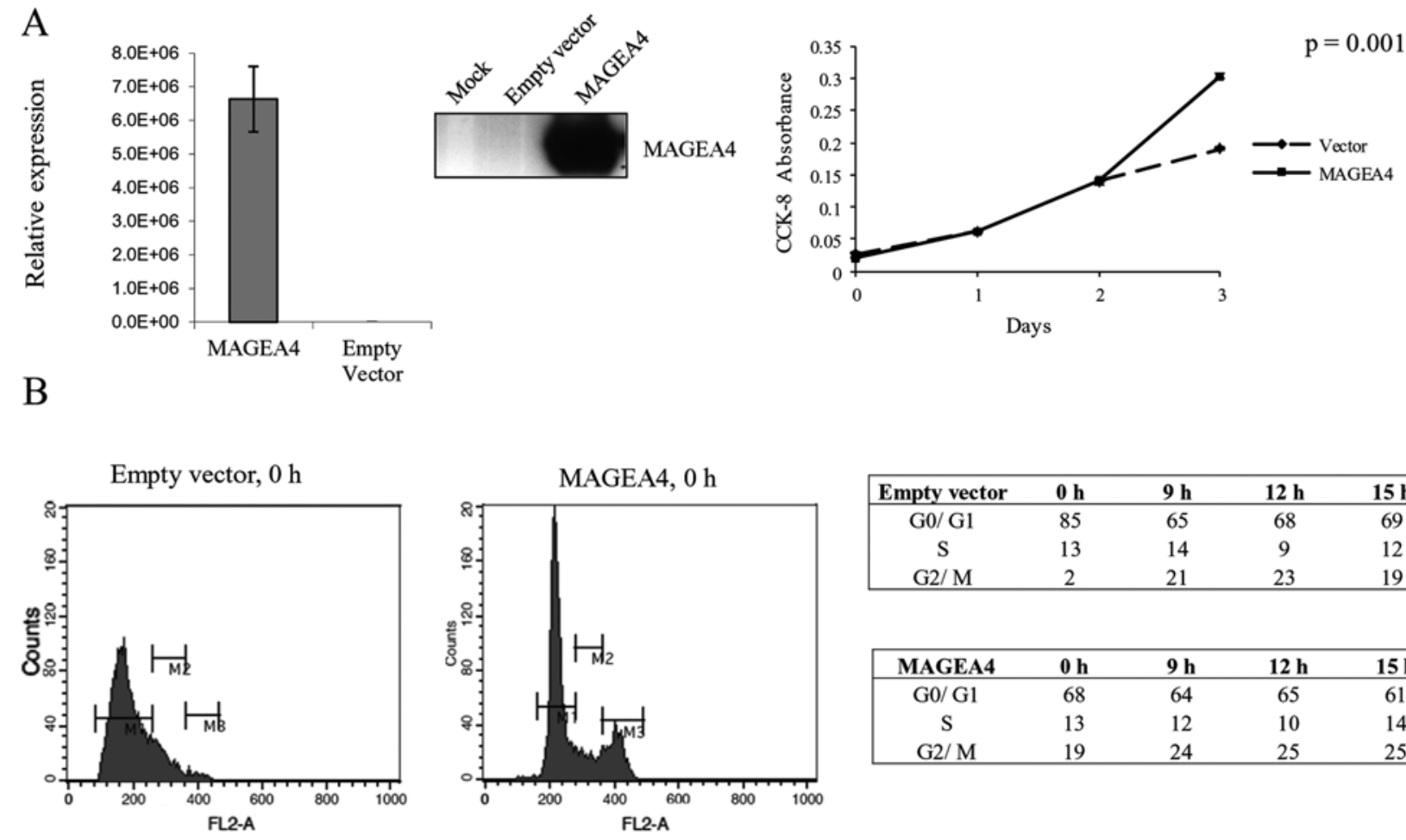

\begin{tabular}{|c|c|c|c|c|}
\hline Empty vector & $0 \mathrm{~h}$ & $9 \mathrm{~h}$ & $12 \mathrm{~h}$ & $15 \mathrm{~h}$ \\
\hline $\mathrm{G} 0 / \mathrm{G} 1$ & 85 & 65 & 68 & 69 \\
\hline $\mathrm{S}$ & 13 & 14 & 9 & 12 \\
\hline $\mathrm{G} 2 / \mathrm{M}$ & 2 & 21 & 23 & 19 \\
\hline MAGEA4 & $\mathbf{O h}$ & $9 \mathrm{~h}$ & $12 \mathrm{~h}$ & $15 \mathrm{~h}$ \\
\hline $\mathrm{G} 0 / \mathrm{Gl}$ & 68 & 64 & 65 & 61 \\
\hline $\mathrm{s}$ & 13 & 12 & 10 & 14 \\
\hline $\mathrm{G} 2 / \mathrm{M}$ & 19 & 24 & 25 & 25 \\
\hline
\end{tabular}

Figure 2. (A) MAGEA4 promotes growth of normal oral keratinocytes. MAGEA4 is overexpressed in NOKSI cells transiently transfected with the MAGEA4 vector compared to control cells transfected with empty vector as shown by qRT-PCR and western blotting. For qRT-PCR expression was normalized to GAPDH. Equal amount of total protein was loaded in all lanes for western blot analysis (mock; untransfected cells). NOK-SI cells overexpressing MAGEA4 show a $37 \%$ increase $(\mathrm{p}=0.001)$ in growth at $72 \mathrm{~h}$ after transfection compared to the control cells transfected with empty vector. (B) MAGEA4 prevents cell cycle arrest. Flow cytometric analysis of DNA content of control and MAGEA4 overexpressing cells at $0 \mathrm{~h}$ after release is shown. M1, cells in G0/G1 phase; M2, cells in S phase; M3, cells in G2/M phase. A significantly lower percentage of cells overexpressing MAGEA4 are arrested in G0 phase (68\%) due to contact inhibition compared to the control cells (85\%). Percentage of cells in different stages of the cell cycle at indicated time points shows that MAGEA4 overexpressing and control cells progressed through the cell cycle similarly.

Monte et al (18) have shown that MAGEA2 recruits HDAC3 to 53 transcription sites to downregulate apoptotic activators, $B A X$ and $C D K N 1 A$. We reasoned that the inhibition of apoptosis caused by MAGEA4 could be a result of downregulation of these p53 target genes. The expression levels of both $B A X$ and CDKNIA were similar in MAGEA4 overexpressing and control cells in absence of G418 (Fig. 4). After treatment with $\mathrm{G} 418$, the expression of both $B A X$ and $C D K N 1 A$ increased in the control cells. However, expression of these 2 genes remained significantly lower in the MAGEA4 overexpressing cells compared to the control cells (Fig. 4). This suggests that MAGEA4 may inhibit apoptosis by reducing the response of p53 targets, $B A X$ and $C D K N 1 A$ to apoptosis inducing agents.

\section{Discussion}

MAGE proteins are members of class I family of CTAs which elicit both cellular and humoral immune responses and thus have been explored as targets for therapeutic cancer vaccines. In the present study, we evaluated the expression of MAGEA4 in a 

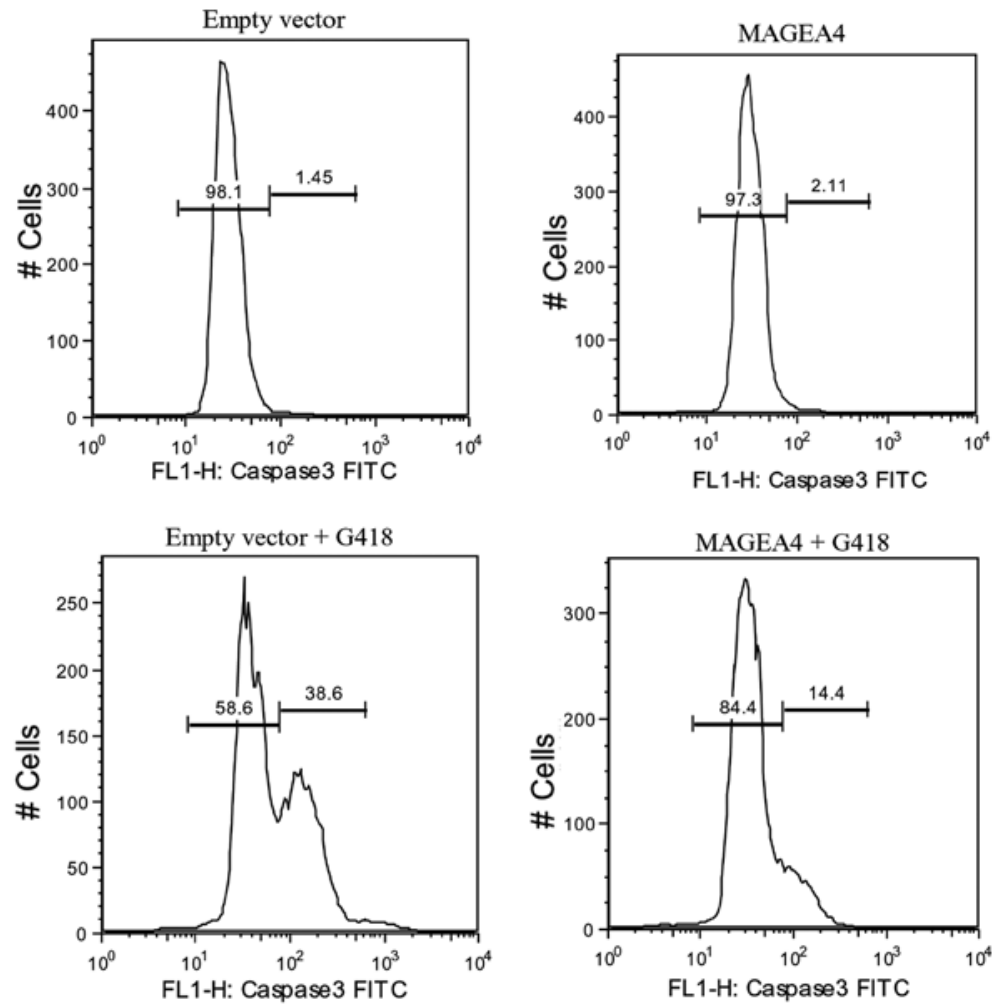

Figure 3. MAGEA4 inhibits apoptosis. Caspase-3 activation, indicative of apoptosis induction, as analyzed by flow cytometry is shown. After $48 \mathrm{~h}$ of treatment with G418, a significantly smaller percentage of MAGEA4 expressing cells (14\%) were positive for active caspase- 3 compared to the control cells transfected with empty vector (39\%). Control cells and cells overexpressing MAGEA4 not treated with G418 were negative for active caspase-3.
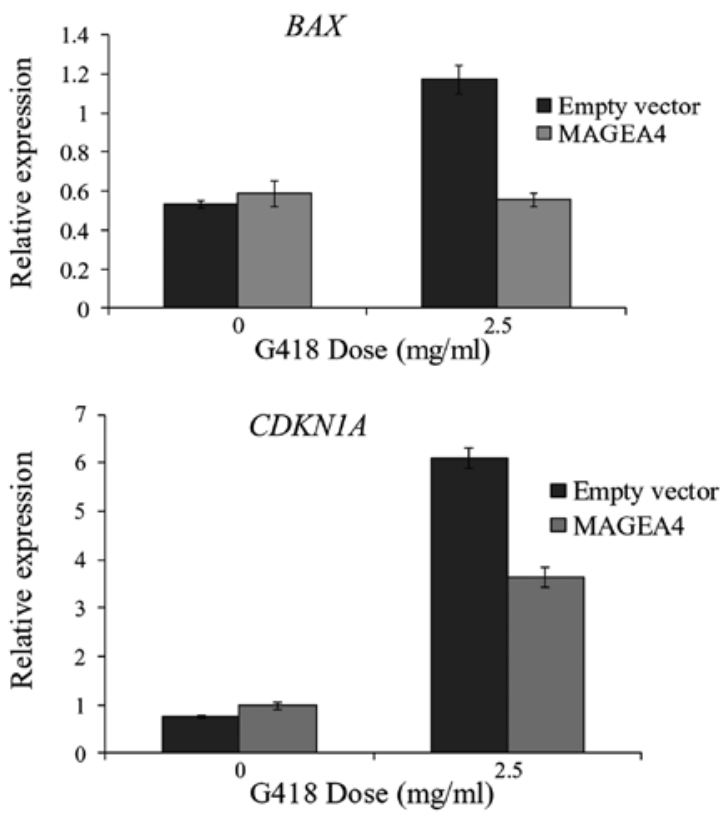

Figure 4. Expression of $B A X$ and $C D K N 1 A$ is reduced in MAGEA4 expressing cells. Control and MAGEA4 overexpressing cells not induced with G418 show similar levels of expression of $B A X$ and $C D K N 1 A$. Induction of apoptosis by G418, increased the expression of these 2 genes in the control cells transfected with empty vector. Expression of these genes remains significantly lower in MAGEA4 overexpressing cells after treatment with G418 for $48 \mathrm{~h}$.

cohort of HNSCC and normal mucosa and found that it is significantly upregulated in tumors. Although it is well established that CTAs are overexpressed in tumors, the biological functions of these proteins remain poorly characterized. Surprisingly, only a few studies have investigated the physiological functions of CTAs in cancers. In order to define the functions of MAGEA4 in HNSCC, we overexpressed MAGEA4 in normal oral keratinocytes and found that it stimulates growth of these cells by inhibiting cell cycle arrest and also making the cells resistant to apoptosis. Of note, MAGEA4 overexpressing cells treated with G418, an inducer of p53-dependent apoptosis, showed lower levels of two p53 target genes, $B A X$ and $C D K N 1 A$. While BAX, a proapoptotic member of the $\mathrm{Bcl}-2$ family proteins, forms pores in the mitochondrial membrane and aids in the release of cytochrome $\mathrm{c}$ into the cytoplasm, CDKN1A has also been reported to enhance apoptosis in response to drug treatment $(25,26)$.

Supporting our results, Yang et al (20) have shown that suppression of the MAGEA genes reduced the viability and also induced apoptosis in three melanoma cell lines. Suppression of apoptosis by MAGE proteins was p53-dependent MAGE knockdown-induced apoptosis in wild-type HCT116 colon cancer cell line but not in $\mathrm{p} 53^{--}$cells. Inoculation of mice with mMAGEB siRNA treated S91 melanoma cells, led to decreased tumor growth compared to S91 cells treated with control siRNA. These results are also supported by observations from another study that melanoma cells expressing high levels of MAGEA proteins are resistant to p53-dependent apoptosis. MAGEA2, specifically, interacts with p53 and recruits transcriptional repressors to $\mathrm{p} 53$ transcription sites thus inhibiting the expression of p53 downstream targets (18). Knockdown of MAGEA and MAGEC2 expression in human mast cells and knockdown of murine MAGEB in mouse mast cells decreases proliferation of these cells (19). Knockdown of these MAGE proteins also 
led to increased apoptosis in these cells. Furthermore, treatment with MAGE siRNA led to decreased tumor growth in a murine model of mastocytosis. MAGEA3 has been found to specifically bind procaspase-12 and render the cells refractory to endoplasmic reticulum stress induced apoptosis (17). All the above studies provide compelling evidence for an oncogenic role for MAGE proteins. However, some contradictory studies have also emerged that support a tumor suppressive role for MAGE proteins. One such study has shown that overexpression of MAGEA4 in human embryonic kidney cells (293 cells) leads to increased apoptosis while knockdown of MAGEA4 in a squamous cell lung cancer cell line H1703 and 293/MAGEA4 cells reduces apoptosis suggesting a tumor suppressive role for MAGEA4 (22). Also, MAGEA4 specifically binds to gankyrin, an oncogene overexpressed in hepatocellular carcinomas, to suppress its oncogenic activity. It suppresses both anchorageindependent growth as well as tumor formation of gankyrin overexpressing cells in athymic nude mice (23).

The MAGEA proteins being highly homologous may be expected to exhibit functional redundancy. However, emerging evidence suggests the involvement of MAGE proteins in a wide spectrum of cellular processes. Thus, in view of the differential roles of MAGEA proteins, it is important to define the functions of individual MAGE proteins in different cancers to better understand their functional significance in tumorigenesis.

\section{Acknowledgements}

This study was based on a web database application provided by Research Information Technology Systems (RITS) - https:// www.rits.onc.jhmi.edu/.

\section{References}

1. Simpson AJ, Caballero OL, Jungbluth A, Chen YT and Old LJ: Cancer/testis antigens, gametogenesis and cancer. Nat Rev Cancer 5: 615-625, 2005.

2. Zendman AJ, Ruiter DJ and Van Muijen GN: Cancer/testisassociated genes: identification, expression profile, and putative function. J Cell Physiol 194: 272-288, 2003.

3. Koslowski M, Bell C, Seitz G, et al: Frequent nonrandom activation of germ-line genes in human cancer. Cancer Res 64: 5988-5993, 2004.

4. Caballero OL and Chen YT: Cancer/testis (CT) antigens: potential targets for immunotherapy. Cancer Sci 100: 2014-2021, 2009.

5. Barker PA and Salehi A: The MAGE proteins: emerging roles in cell cycle progression, apoptosis, and neurogenetic disease. J Neurosci Res 67: 705-712, 2002.

6. Sugita M, Geraci M, Gao B, et al: Combined use of oligonucleotide and tissue microarrays identifies cancer/testis antigens as biomarkers in lung carcinoma. Cancer Res 62: 3971-3979, 2002.

7. De Smet C, De Backer O, Faraoni I, Lurquin C, Brasseur F and Boon T: The activation of human gene MAGE-1 in tumor cells is correlated with genome-wide demethylation. Proc Natl Acad Sci USA 93: 7149-7153, 1996.
8. De Smet C, Lurquin C, Lethe B, Martelange V and Boon T: DNA methylation is the primary silencing mechanism for a set of germ line- and tumor-specific genes with a CpG-rich promoter. Mol Cell Biol 19: 7327-7335, 1999.

9. Weber J, Salgaller M, Samid D, et al: Expression of the MAGE-1 tumor antigen is up-regulated by the demethylating agent 5-aza2'-deoxycytidine. Cancer Res 54: 1766-1771, 1994.

10. Glazer CA, Smith IM, Ochs MF, et al: Integrative discovery of epigenetically derepressed cancer testis antigens in NSCLC. PloS One 4: e8189, 2009.

11. Smith IM, Glazer CA, Mithani SK, et al: Coordinated activation of candidate proto-oncogenes and cancer testes antigens via promoter demethylation in head and neck cancer and lung cancer. PloS One 4: e4961, 2009.

12. Bhan S, Negi SS, Shao C, et al: BORIS binding to the promoters of cancer testis antigens, MAGEA2, MAGEA3, and MAGEA4, is associated with their transcriptional activation in lung cancer. Clin Cancer Res 17: 4267-4276, 2011.

13. Vatolin S, Abdullaev Z, Pack SD, et al: Conditional expression of the CTCF-paralogous transcriptional factor BORIS in normal cells results in demethylation and derepression of MAGE-A1 and reactivation of other cancer-testis genes. Cancer Res 65: 7751-7762, 2005

14. Hong JA, Kang Y, Abdullaev Z, et al: Reciprocal binding of CTCF and BORIS to the NY-ESO-1 promoter coincides with derepression of this cancer-testis gene in lung cancer cells. Cancer Res 65: 7763-7774, 2005.

15. Scanlan MJ, Gure AO, Jungbluth AA, Old LJ and Chen YT: Cancer/testis antigens: an expanding family of targets for cancer immunotherapy. Immunol Rev 188: 22-32, 2002.

16. Ghafouri-Fard S and Modarressi MH: Cancer-testis antigens: potential targets for cancer immunotherapy. Arch Iran Med 12: 395-404, 2009

17. Morishima N, Nakanishi K, Takenouchi H, Shibata T and Yasuhiko Y: An endoplasmic reticulum stress-specific caspase cascade in apoptosis. Cytochrome c-independent activation of caspase-9 by caspase-12. J Biol Chem 277: 34287-34294, 2002.

18. Monte M, Simonatto M, Peche LY, et al: MAGE-A tumor antigens target p53 transactivation function through histone deacetylase recruitment and confer resistance to chemotherapeutic agents. Proc Natl Acad Sci USA 103: 11160-11165, 2006.

19. Yang B, O'Herrin S, Wu J, Reagan-Shaw S, Ma Y, Nihal M and Longley BJ: Select cancer testes antigens of the MAGE-A, -B, and $-\mathrm{C}$ families are expressed in mast cell lines and promote cell viability in vitro and in vivo. J Invest Dermatol 127: 267-275, 2007.

20. Yang B, O'Herrin SM, Wu J, et al: MAGE-A, mMage-B, and MAGE-C proteins form complexes with KAP1 and suppress p53-dependent apoptosis in MAGE-positive cell lines. Cancer Res 67: 9954-9962, 2007.

21. Glazer CA, Smith IM, Bhan S, et al: The role of MAGEA2 in head and neck cancer. Arch Otolaryngol Head Neck Surg 137: 286-293, 2011.

22. Peikert T, Specks U, Farver C, Erzurum SC and Comhair SA: Melanoma antigen A4 is expressed in non-small cell lung cancers and promotes apoptosis. Cancer Res 66: 4693-4700, 2006.

23. Nagao T, Higashitsuji $\mathrm{H}$, Nonoguchi $\mathrm{K}$, et al: MAGE-A4 interacts with the liver oncoprotein gankyrin and suppresses its tumorigenic activity. J Biol Chem 278: 10668-10674, 2003.

24. Jin QH, Zhao B and Zhang XJ: Cytochrome c release and endoplasmic reticulum stress are involved in caspase-dependent apoptosis induced by G418. Cell Mol Life Sci 61: 1816-1825, 2004.

25. Kuribayashi K and El Deiry WS: Regulation of programmed cell death by the p53 pathway. Adv Exp Med Biol 615: 201-221, 2008.

26. Lincet H, Poulain L, Remy JS, Deslandes E, Duigou F, Gauduchon P and Staedel C: The p21(cip1/waf1) cyclin-dependent kinase inhibitor enhances the cytotoxic effect of cisplatin in human ovarian carcinoma cells. Cancer Lett 161: 17-26, 2000. 